\title{
Automatic Face Recognition System using Pattern Recognition Techniques: A Survey
}

\author{
Ningthoujam Sunita Devi \\ Department of Computer Science \\ Assam University, Silchar-788011
}

\author{
Prof.K.Hemachandran \\ Department of Computer Science \\ Assam University, Silchar-788011
}

\begin{abstract}
The Automatic Face Recognition system is widely applied in new technologies. This system works beyond the ability of human vision. The limited vision of human eye in identifying vast number of human faces is overcome by the automatic face recognition with many more advantages. The basic purpose of face recognition system is to compare the image video which is stored in a database with the image video in real time variation. Many techniques have been used in face recognition system. This paper present a survey of several techniques used in face recognition system, an approach to the detection and identification of human face.
\end{abstract}

\section{Keywords}

Face recognition, face detection, face extraction.

\section{INTRODUCTION}

Automatic Face recognition is one of the biometrics that is being used for the last 50 years or so. The early face recognition systems required the intervention of a human to locate the features, such as eyes, ears, nose and mouth, on the photograph image before the calculation of distance and ratio parameters with respect to a common reference point.

Face recognition is typically used in security system and can be compared to other biometrics such as fingerprint or iris recognition system. Facial recognition research and Fingerprint Recognition Technology (FRT) are the subfields, in a larger field of pattern recognition research and technology. Pattern recognition technology uses statistical techniques to detect and extract patterns from data in order to match it with patterns stored in a database. The data upon which the recognition system works (such as an image of a face) is no more than a set of pixel-level patterns for the system, that is, the pattern recognition system does not perceive meaningful "faces" as a human would understand them. Nevertheless, it is very important for these systems to be able to locate or detect a face in a field of vision so that it is only the image pattern of the face (and not the background "noise") that is processed and analyzed. Facial expressions are the facial changes in response to a person's internal emotional states, intentions or social communications. As one of the most successful applications of image analysis and understanding, face recognition has recently received significant attention, especially during the past several years. Even though current machine recognition systems have reached a certain level of maturity, their success is limited by the conditions imposed by many real applications.

The purpose of face recognition is mainly to identify a person, as a key to security (Biometric face recognition technology) and a wide variety of applications like law enforcement. As compared with the other biometric systems like finger print, palm print and iris, the face recognition has distinct advantage, because of its non-contact process. Face image can be captured from a distance without touching the person being identified, and the identification does not require interaction with the person. In addition, face recognition serves the crime deterrent purpose because facial images that have been recorded and archived can later be used to identify a person. The goal of face reorganization is to implement the system for a particular face and distinguish it from a large number of stored faces with some real-time variations [2]. The generic face recognition system is shown in fig: 1 .

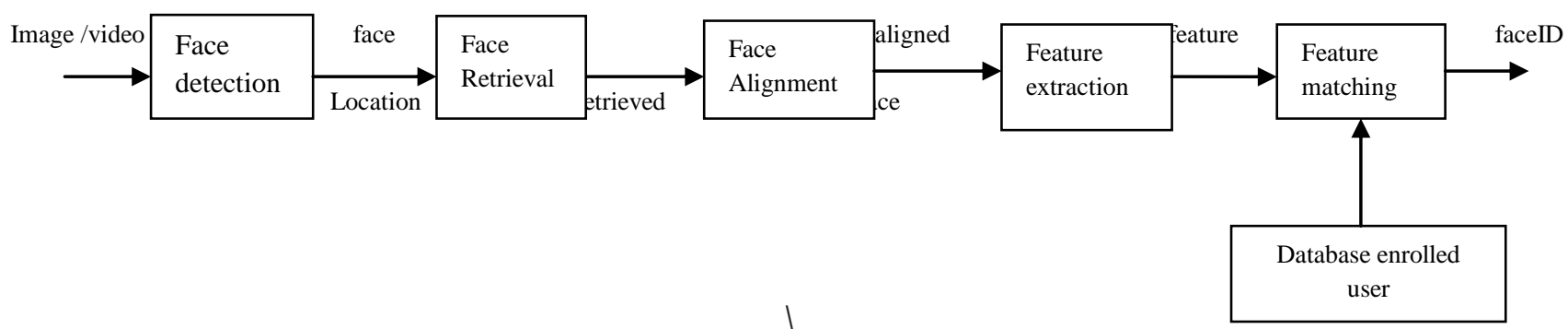

Fig.1. Face recognition processing flow

\section{SOME EXISTING TECHNIQUES FOR FACE RECOGNITION}

A number of methods have been proposed in the area of automatic face recognition. Face recognition systems broadly based on two approaches:1) holistic approach and 2)local approach as shown in fig 2. Some of the existing methods under different categories are eigen faces also called principal component analysis (PCA) [3], Fisher faces also called Fisher Discriminates Analysis (FDA), linear discriminates analysis (LDA) [5], self organizing map and convolution network [8,10], line edge maps (LEMs) [13], directional corner point (DCP) [20], template matching [14], modular PCA [15], elastic bunch graph matching (EBGM) [17], and local binary patterns (LBP) [19]. 
Table 1 Categorisation of face recognition technique

\begin{tabular}{|c|c|}
\hline \multicolumn{2}{|c|}{ Face Recognition System } \\
\hline Holistic Approach & Local Approach \\
\hline $\begin{array}{c}\text { Principal Component } \\
\text { Analysis(PCA) }\end{array}$ & Templates Matching \\
\hline $\begin{array}{c}\text { Fisher Discriminate } \\
\text { Analysis(FDA) }\end{array}$ & Modular PCA \\
\hline $\begin{array}{c}\text { Linear Discriminates } \\
\text { Analysis(LDA) }\end{array}$ & Elastic bunch graph matching \\
\hline $\begin{array}{c}\text { Self Organizing map and } \\
\text { Convolution Network }\end{array}$ & Local Binary Patterns \\
\hline $\begin{array}{c}\text { Line Edges Map(LEM) } \\
\text { Directional Corner Point(DCP) }\end{array}$ & $* * * * * * *$ \\
\hline
\end{tabular}

\subsection{Holistic approaches:}

Kirby and Sirovich [3] have used the principal component analysis to efficiently represent face images by a small number of coefficients corresponding to the most significant Eigen values. Turk and Pentland [4] have been utilized eigen faces for face detection and identification. In particular, a set of eigen vectors and eigen values were first calculated through principal component analysis to form the eigen space of human faces (or "Eigen faces") from a training face image set. The gallery and probe images were projected to these eigen space and their eigen values are compared in the recognition stage. The performance increase of eigen face was from $61.4 \%$ to $89.5 \%$. This kind of increase is due to the capability for face recognition algorithm of tolerating small pose variation.

The Eigen faces approach appears to be a fast, simple, and practical method, which has become the most widely used face recognition technique. However, it does not provide invariance over changes in poses and scales. Belhumeur and Hespanha [6] also examine LDA, a representation obtained through supervised learning on the second-order image statistics. LDA (or Fisher Discriminant Analysis) is a statistical approach based on the same statistical principles as PCA. This technique finds the underlying vectors in the facial feature spaces (Vectors) that would maximize the variance between individuals (or classes) and minimize the variance within a number of samples of the same person. Using this specific projection method, the training and recognition were performed similarly to those of Eigen faces. To overcome the problem of within-class scatter matrix being singular, the face images were first projected using PCA to reduce the dimensionality to a lower level that FDA can handle. In this case, it requires multiple gallery images per class (person) otherwise FDA will be identical to PCA. In face recognition, when dealing with high dimensional image data, the class scatter matrix $S_{w}\left(S_{w}=\Sigma \Sigma M c(x-m c)(x-m c) T\right)$ is often singular. To overcome this problem, PCA is first used with the sample data to reduce its dimensionality, Vinoth, M., et al proposed an analysis called PCLDA [7]. A holistic face recognition approaches, both FDA and PCA are very sensitive to pose variations, because in depth rotations of $3 \mathrm{D}$ human faces misalignment of image pixels usually occur which are the only classification clues for these holistic approaches. One of the first artificial neural network techniques used for face recognition is the single layer network WISARD, which contains a separate network for each stored individual. Lin et al. [7] used a probabilistic decision-based neural network (PDBNN) which also used one network for one face and required multiple gallery images per person in training the network. Lawrence et al. [10] proposed a hybrid neural network, which combined local image sampling, a selforganizing map (SOM), and a convolution network $(\mathrm{CN})$. Dinesh K. et al. $[8,11]$ used the Self-Organizing Map (SOM), an unsupervised learning process, to learn the distribution of a set of patterns without any class information. It has the property of topology preservation. There is a competition among the neurons to be activated or fired. The result is that only one neuron that wins the competition is fired and is called winner-takes all neuron. SOMs may be onedimensional, two-dimensional or multidimensional, but the most common ones are either one-dimensional or twodimensional maps. The number of input connections depends on the number of attributes to be used in the classification The neuron with weights closest to the input data vector is declared the winner during the training. Then the weights of all of the neurons in the neighborhood of the winning neuron are adjusted by an amount inversely proportional to the distance. It clusters and classifies the data set based on the set of attributes used. For feature detection and classification stage, a convolution network has been applied which contains iterative convolution and down-sampling layers. And the main achievement of SOM is its high-speed processing capability and low computational requirement in terms of both speed and memory utilization.

Gao et.al. [13] has proposed line edge map (LEM) which gives a distance measurement between two line edge maps of faces and performs face matching based on those measures. The LEM of a face image is generated by sequentially (1) extracting edges, (2) thinning, and (3) polygonal line fitting. To measure the similarity between two LEMs, a line segment Hausdorff distance was introduced, which computes the distance two line segments as root-sum-square of three distance components, i.e., parallel distance, orientation distance, and perpendicular distance. In recognition, each face image was first converted to an LEM, followed by matching probe LEMs against gallery LEMs using the line segment Hausdorff distance. Line edge map achieved $92 \%$ accuracy in their experiment. A new face feature descriptor, directional corner points (DCPs) [20], is proposed to integrate the structural connectivity information with spatial features of a face image. A DCP is represented by its Cartesian coordinates and two directional attributes pointing to the points anterior and posterior neighboring corner points. The distance of two DCPs is measured by calculating the warping cost through translation, rotation and opening/closing operations and averaging the minimum warping costs as the dissimilarity score. Face image retrieval using DCPs is generally economical for storage and robust to illumination changes. Its robustness to illumination changes is inherited from edge maps, because a corner point can be considered as the "edge of edges".

After studying the above mentioned holistic techniques we have summarized their respective advantages and disadvantages in the following table 2. 
Table 2The advantages and disadvantages of the holistic approach

\begin{tabular}{|l|l|l|}
\hline Approach & \multicolumn{1}{|c|}{ Advantage } & \multicolumn{2}{|c|}{ Disadvantage } \\
\hline $\begin{array}{l}\text { Eigen faces } \\
{[2]}\end{array}$ & Simple, fast & $\begin{array}{l}\text { Sensitive to pixel } \\
\text { misalignment, cannot } \\
\text { separate image } \\
\text { variances }\end{array}$ \\
\hline $\begin{array}{l}\text { Fisher faces } \\
{[4]}\end{array}$ & $\begin{array}{l}\text { Maximize the } \\
\text { separability of } \\
\text { different identities }\end{array}$ & $\begin{array}{l}\text { Sensitive to pixel } \\
\text { misalignment, linear } \\
\text { classes cannot } \\
\text { adequately describe } \\
\text { pose variations }\end{array}$ \\
\hline $\begin{array}{l}\text { SOM+CN } \\
{[6,8]}\end{array}$ & $\begin{array}{l}\text { Fast, tolerance to } \\
\text { pixel misalignment } \\
\text { due to quantization }\end{array}$ & $\begin{array}{l}\text { Linear mapping } \\
\text { cannot adequately } \\
\text { describe pose } \\
\text { variations }\end{array}$ \\
\hline LEM [11] & $\begin{array}{l}\text { Simple, no training } \\
\text { and facial } \\
\text { component } \\
\text { detection required }\end{array}$ & $\begin{array}{l}\text { Sensitive to edge } \\
\text { distortions caused by } \\
\text { pose variation }\end{array}$ \\
\hline DCP [17] & $\begin{array}{l}\text { Fast, no training } \\
\text { and facial } \\
\text { component } \\
\text { detection required }\end{array}$ & $\begin{array}{l}\text { Sensitive to edge } \\
\text { distortions caused by } \\
\text { pose variation }\end{array}$ \\
\hline
\end{tabular}

\subsection{Local Approach:}

In local approaches, a set of isolated points or region on the face images are mostly involved and the classification patterns are obtained from a limited region in the face image. Bruneli and Poggio [12] selected a set of 4 feature templates, i.e., the eyes, nose, mouth, and the whole face, for all of the available faces. These feature templates are oftenly used for the identification of the printed character, number and other small, simple object. These feature templates from the input image are compared with those of the image which are stored in database in the same region along the normalized cross matching. Summed matching scores were used for resolving the recognition process. During the process, recognition system has to be tolerant to certain discrepancies between gallery and probe images, this tolerance might average out the differences that make individual faces unique. So, this is the drawback of templates matching. Gottmukkal and Asari [13] extended PCA to modular PCA to improve robustness of face recognition. In this type of method, the face images are split up into smaller parts as a sub-images and the PCA approach is applied for each and every sub-images. However, the MPCA technique does not simply apply PCA in each independent face region; since it keeps the relation between the regions and the global information of the face. MPCA establishes multiple Eigen spaces around facial components (e.g., eyes, nose, and mouth) to form "Eigen features" in place of building a holistic Eigen space for the entire images. (Fig. 2).

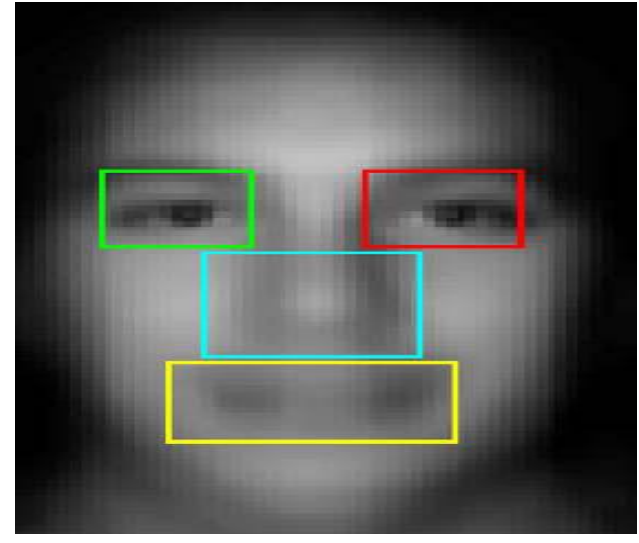

Fig 2. The modular PCA builds multiple eigen spaces

(Eigen features) in the regions of facial components (e.g., eyes, nose, and mouth) to achieve pose tolerances.

One of the most effective local face recognition methods is elastic bunch graph matching [16]. In this method, human faces were described using Gabor wavelets in facial components (e.g., eyes, nose, and mouth) and an extended dynamic link architecture (DLA) [14] for graph matching. In feature extraction, a Gabor jet on a point of a face image was introduced as a set of 40 Gabor wavelet coefficients obtained by convoluting 40 Gabor kernels with the local region around the point. In the recognition process, the Gabor features were extracted from the facial components and the gallery images were compared with the probe images by calculating the similarity of the two sets of Gabor jets. In spite of expensive computation, EBGM perform better than holistic approaches on the testing sets containing in-depth pose variations, which is mainly due to Gabor features' robustness against image distortion and scaling [15]. In [16], elastic graph matching hold forth and made some changes to apply a further Fourier transform on Gabor wavelet coefficients to be used as features and to perform classifications using kernel- based projection discriminative analysis (KPDA) to achieve pose and expression tolerance.

Ahonen et al. $[18,20]$ used local binary patterns which is a successful texture descriptor and applied to the task of face recognition. The local binary pattern is obtained by binarising the gradients of centre point to its 8 neighbouring points pixelwisely and this binary pattern is used as image features for classification. Then the face image is distinguish into several sub-regions (or patches) and within each and every patches, the histogram of the local pixel-wise patterns is calculated. The histograms are compared along the calculating weighted Chi square distance, whose weights are trained by separate recognition process on a single patch, while comparing the images. After studying the above mentioned local techniques we have summarized their respective advantages and disadvantages in the following table 3.

Table 3 The advantages and disadvantages of the local approach

\begin{tabular}{|l|l|l|}
\hline Approach & Advantage & Disadvantage \\
\hline Template & Simple, local & Sensitive to pixel \\
matching [12] & regions around & misalignment in sub- \\
& $\begin{array}{l}\text { facial components } \\
\text { provide some } \\
\text { tolerance to pose } \\
\text { variations }\end{array}$ & $\begin{array}{l}\text { dependent on facial } \\
\text { component detection }\end{array}$ \\
\hline
\end{tabular}




\begin{tabular}{|l|lr|l|}
\hline Modular PCA & $\begin{array}{l}\text { Simple, rocal } \\
\text { regions around } \\
\text { facial components } \\
\text { provider some } \\
\text { tolerance to pose } \\
\text { variations }\end{array}$ & $\begin{array}{l}\text { Sensitive to pixel } \\
\text { misalignment in sub- } \\
\text { image regions, } \\
\text { dependent on facial } \\
\text { component detection }\end{array}$ \\
\hline EBGM [15] & $\begin{array}{l}\text { Local regions } \\
\text { around racial } \\
\text { components and } \\
\text { Gabor r wavelet } \\
\text { provider pose } \\
\text { tolerance }\end{array}$ & $\begin{array}{l}\text { Slow, distortions } \\
\text { within local regions } \\
\text { were not treated }\end{array}$ \\
\hline LBP [18] & $\begin{array}{l}\text { Simple, histogram } \\
\text { in local regions } \\
\text { tolerates pixel } \\
\text { misalignment }\end{array}$ & $\begin{array}{l}\text { Image division is } \\
\text { problematic when } \\
\text { pose variation is } \\
\text { large }\end{array}$ \\
\hline
\end{tabular}

\section{CONCLUSIONS}

In this research paper we tried to cover the details of Face recognition system using Pattern Recognition methods. We have presented a short description of the various Face Recognition Systems. There are various techniques available for Face Recognition but from the above discussion we can say that if we apply Neural Network in face Recognition system we can achieve better recognition rate than the existing classical techniques

\section{ACKNOWLEDGMENT:}

Ningthoujam Sunita Devi gratefully acknowledges UGC for the award of UGC fellowship for the research work.

\section{REFERENCES}

[1] A.J.Goldstein, L.D Harmon and A.B Lesk "Identification of human Face," Proc .IEEE, May 1971, vol.59, No.5, pp. 748-760.

[2] Pandya, Jigar M., Devang Rathod, and Jigna J. Jadav. "A Survey of Face Recognition approach." International Journal of Engineering Research and Applications (IJERA) 3.1 (2013): 632-635.

[3] M. Kirby, L. Sirovich, Application of the KarhunenLoève procedure for the characterization of human face, IEEE transactions on pattern analysis and machine intelligence. vol. 12, January 1990.

[4] M.A. Turk, A.P. Pentland, Face recognition using eigen faces, in: Proceedings of the IEEE Conference on CVPR, 1991, pp. 586-591.

[5] Lu, J., Plataniotis Kostantinos, N., Venetsanopoulos Anastasios, N., 2003. Face recognition using LDA-based algorithms. IEEE Trans.Neural Networks Vol.14, No (1), pp. 195-200.

[6] P.N. Belhumeur, J.P. Hespanha, D.J. Kriegman, Eigen faces vs. Fisher faces: recognition using class specific linear projection, IEEE Transactions On Pattern Analysis and Machine Intelligence, Vol. 19, No. 7, July 1997,pp. 711-720.

[7] Vinoth, M., et al. "Recognition Based System Using Principal Component Linear Discriminant Analysis." methods 2.1 (2012).

[8] Dinesh Kumar , C.S.Rai and Shakti Kumar, ' Face Recognition Using Self-Organizing Map and Principal Component Analysis' 2005 IEEE,pp.1469-1473.
[9] S.-H. Lin, S.-Y. Kung, L.-J. Lin, Face recognition/detection by probabilistic decision-based neural network, IEEE Trans. Neural NetworkVol. 8, No. (1) (1997), pp. 114-132.

[10] Lawrence, Richard D., George S. Almasi, and Holly E. Rushmeier. "A scalable parallel algorithm for selforganizing maps with applications to sparse data mining problems." Data Mining and Knowledge Discovery 3.2 (1999): 171-195.

[11] Ghorpade, Santaji, Jayshree Ghorpade, and Shamla Mantri,Patter Recognition using Neural Networks, International Journal of Computer Science and Technology (IJCST) 2.6 (2010)pp.92-98.

[12] A. Pentland, B. Moghaddam, T. Starner, View-based and modular eigenspaces for face recognition, in: Proceedings of the IEEE Conference on CVPR, 1994, pp. 84-91.

[13] Y. Gao, M.K.H. Leung, Face recognition using line edge map, IEEE Transactions On Pattern Analysis And Machine Intelligence, Vol. 24, No. 6, June (2002),pp. 764-779.

[14] R. Brunelli, T. Poggio, Face recognition: features versus templates, IEEE Transactions on Pattern Analysis and Machine Intelligence, Vol. 15, No. 10, October (1993), pp. 1042-1052.

[15] R. Gottmukkal and V. K. Asari, "An improved face recognition technique based on modular PCA approach," Pattern Recognition Letters, Vol.24, No.4, pp. 429-436, 2004.

[16] M. Lades, J.C. Vorbruggen, J. Buhmann, J. Lange, C. Vander Malsburg, R.P. Wurtz, W. Konen, Distortion invariant object recognition in the dynamic link architecture, IEEE Trans. Comput. Vol. 42 (1993), PP. 300-311.

[17] D.Aradhana, H.Girish, K.Karibasappa and C.Reddy, Face recognition by elastic bunch graph method using a group based adaptive tolerant neural network, Vol.1, No. (4) (2001), pp. 194-203.

[18] H.C. Shin, J.H. Park, S.D. Kim, Combination of warping robust elastic graph matching and kernel-based projection discriminate analysis for face recognition, IEEE Trans. Multimedia 9 (6) (2007),pp. 1125-1136.

[19] T. Ahonen, A. Hadid, M. Pietikainen, Face description with local binary patterns: application to face recognition IEEE Transactions On Pattern Analysis And Machine Intelligence, Vol. 28, No. 12, December 2006,pp. 20372041.

[20] Gao, Yongsheng, and Yutao Qi. "Robust visual similarity retrieval in single model faces databases." Pattern Recognition 38.7 (2005), pp. 1009-1020.

[21] T. Ojala, M. Pietikainen, T. Maenpaa, Multi resolution gray-scale and rotation invariant texture classification with local binary patterns, IEEE Trans. Pattern Anal. Mach. Intell.vol.24,No (7) (2002) ,pp.971-987.

[22] Huang, Di, et al. "Local binary patterns and its application to facial image analysis: a survey." Systems, Man, and Cybernetics, Part C: Applications and Reviews, IEEE Transactions on 41.6 (2011): 765-781. 